\title{
THE EFFECT OF DIFFUSION ON CRITICAL QUASILINEAR ELLIPTIC PROBLEMS
}

\author{
Renato José de Moura - Marcos Montenegro
}

Abstract. We discuss the role of the diffusion coefficient $a(x)$ on the existence of a positive solution for the quasilinear elliptic problem involving critical exponent

$$
\begin{cases}-\operatorname{div}\left(a(x)|\nabla u|^{p-2} \nabla u\right)=u^{p^{*}-1}+\lambda u^{p-1} & \text { in } \Omega, \\ u=0 & \text { on } \partial \Omega,\end{cases}
$$

where $\Omega$ is a smooth bounded domain in $\mathbb{R}^{n}, n \geq 2,1<p<n, p^{*}=$ $n p /(n-p)$ is the critical exponent from the viewpoint of Sobolev embedding, $\lambda$ is a real parameter and $a: \bar{\Omega} \rightarrow \mathbb{R}$ is a positive continuous function. We prove that if the function $a(x)$ has an interior global minimum point $x_{0}$ of order $\sigma$, then the range of values $\lambda$ for which the problem above has a positive solution relies strongly on $\sigma$. We discover in particular that the picture changes drastically from $\sigma>p$ to $\sigma \leq p$. Some sharp answers are also provided.

\section{Introduction and main theorems}

In the 80 's decade, Brezis and Nirenberg investigated, in the celebrated paper [9], the existence of a positive solution for the problem

$$
\begin{cases}-\Delta u=u^{(n+2) /(n-2)}+\lambda u & \text { in } \Omega, \\ u=0 & \text { on } \partial \Omega,\end{cases}
$$

2010 Mathematics Subject Classification. 35B33, 35J60, 49K20, 76R50.

Key words and phrases. Non-uniformly elliptic operators, critical Sobolev exponent, best constant, Hardy-Sobolev inequality. 
on a smooth bounded domain $\Omega \subset \mathbb{R}^{n}, n \geq 3$, where $\lambda$ is a real parameter. As it is well known, the existence of such a positive solution relies on the value of the constant $\lambda$ compared with the first eigenvalue $\lambda_{1}(-\Delta)$ corresponding to the Laplace operator on $\Omega$ under Dirichlet boundary condition. According to one of the main results of [9], the problem (1.1) admits a positive solution if, and only if, $\lambda \in\left(0, \lambda_{1}(-\Delta)\right)$ provided that $n \geq 4$ and $\Omega$ is star-shaped. Besides, when $n=3$ and $\Omega$ is a ball, a positive solution of (1.1) exists if, and only if, $\lambda \in\left(\lambda_{1}(-\Delta) / 4, \lambda_{1}(-\Delta)\right)$. Since then, a lot of attention has been devoted to various questions and extensions related to (1.1). We refer for instance to the Struwe's [36] and Willem's [38] books and references therein for an overview on the so-called Brezis-Nirenberg problem.

A closely related extension which has been widely addressed in the literature is the existence of a positive solution for the problem

$$
\begin{cases}-\Delta_{p} u=|u|^{p^{*}-2} u+\lambda|u|^{p-2} u & \text { in } \Omega \\ u=0 & \text { on } \partial \Omega\end{cases}
$$

where $n \geq 2,1<p<n, \Delta_{p} u=\operatorname{div}\left(|\nabla u|^{p-2} \nabla u\right)$ represents the $p$-Laplace operator and $p^{*}=n p /(n-p)$ denotes the critical Sobolev exponent to the embedding of $W_{0}^{1, p}(\Omega)$ into Lebesgue spaces. Again the existence of a positive solution for (1.2) relies on the location of $\lambda$ with respect to the first eigenvalue $\lambda_{1}\left(-\Delta_{p}\right)$ corresponding to the $p$-Laplace operator on $\Omega$ under Dirichlet boundary condition. The above-mentioned result of [9] valid for $n \geq 4$ and star-shaped domains has been extended by Egnell [19], Garcia Azorero and Peral Alonso [21] and Guedda and Veron [25], who have proved that the problem (1.2) admits a positive solution if, and only if, $\lambda \in\left(0, \lambda_{1}\left(-\Delta_{p}\right)\right)$ provided that $1<p \leq$ $\sqrt{n}$. Under this range on $p$, De Valerioli and Willem [14] has also discussed the existence of solution. The case $p>\sqrt{n}$ has been considered by Egnell [19], who has found a number $\lambda^{*}$ so that a positive solution of (1.2) exists for any $\lambda \in\left(\lambda^{*}, \lambda_{1}\left(-\Delta_{p}\right)\right)$. When $\lambda \geq \lambda_{1}\left(-\Delta_{p}\right)$, results on existence of a nontrivial solution have been established in [11] and [22] for $p=2$ and in [2] and [15] for $p \neq 2$.

Another important line of investigation consists of the study of existence of positive (or nontrivial) solutions for problems connected to (1.2) in the presence of diffusion such as

$$
\begin{cases}-\Delta_{p} u=\alpha(x) u^{p^{*}-1}+\beta(x) u^{p-1} & \text { in } \Omega \\ u=0 & \text { on } \partial \Omega\end{cases}
$$

and

$$
\begin{cases}-\operatorname{div}\left(a(x)|\nabla u|^{p-2} \nabla u\right)=u^{p^{*}-1}+\lambda u^{p-1} & \text { in } \Omega, \\ u=0 & \text { on } \partial \Omega\end{cases}
$$


where the diffusion coefficients $\alpha(x), \beta(x)$ and $a(x)$ are positive continuous functions on $\bar{\Omega}$. The problem (1.3) has been focused in various works in the case $p=2$ as, for instance, [5], [7], [11], [20], [22]-[24], [32], [33], [40] and in the case $p \neq 2$, in [2], [6], [15], [17], [19], [28], among others. Already the problem (1.4) has been investigated in a few works where a comprehensive study has been performed, namely [18], [26] and [31] for $p=2$ and [19] for $p \neq 2$.

Our main goal is discussing the effect of the order of global minimum points of the diffusion $a(x)$ on the existence of a positive solution for the problem (1.4) when $p \neq 2$.

For simplicity of notation, let $\mathcal{L}_{p, a} u=\operatorname{div}\left(a(x)|\nabla u|^{p-2} \nabla u\right)$. The operator $\mathcal{L}_{p, a}$ is degenerate elliptic for $p>2$ and singular for $p<2$. By a positive solution of (1.4) we mean a nontrivial nonnegative weak solution in $W_{0}^{1, p}(\Omega)$. A positive solution in $C^{1}(\bar{\Omega})$ will be simply referred as a positive $C^{1}$ solution.

Let $\lambda_{1}\left(-\mathcal{L}_{p, a}\right)$ be the first eigenvalue corresponding to the problem

$$
\begin{cases}-\mathcal{L}_{p, a} u=\lambda|u|^{p-2} u & \text { in } \Omega, \\ u=0 & \text { on } \partial \Omega .\end{cases}
$$

A fairly simple fact that deserves mention due to Egnell [19] is the existence of a number $\lambda^{*}$ less than $\lambda_{1}\left(-\mathcal{L}_{p, a}\right)$ such that a positive solution of (1.4) exists for any $\lambda \in\left(\lambda^{*}, \lambda_{1}\left(-\mathcal{L}_{p, a}\right)\right)$. We are interested in the seeking for optimal values of $\lambda^{*}$ or, at least, for good upper and lower bounds of $\lambda^{*}$ depending of the order of global minimum points of the function $a(x)$.

We first state two main existence results, the first one to higher order minimum points and the second one dealing with lower order.

TheOrem 1.1. Assume that $a(x)$ attains its minimum value at $x_{0} \in \Omega$ and there exist constants $\sigma>p$ and $C_{0}>0$ such that

$$
a(x) \leq a\left(x_{0}\right)+C_{0}\left|x-x_{0}\right|^{\sigma}
$$

around $x_{0}$. If $1<p \leq \sqrt{n}$, then the problem (1.4) has a positive solution for any $\lambda \in\left(0, \lambda_{1}\left(-\mathcal{L}_{p, a}\right)\right)$.

The conclusion of Theorem 1.1 remains indeed valid if the condition (1.6) is replaced by $a(x)=a\left(x_{0}\right)+o\left(\left|x-x_{0}\right|^{p}\right)$ around $x_{0}$, see Section 3 for full details. The importance of working with global minimum is justified by Lemma 2.1.

TheOREm 1.2. Assume that there exist $x_{0} \in \Omega$ and constants $0<\sigma \leq p$ and $C_{0}>0$ such that

$$
a(x) \geq a\left(x_{0}\right)+C_{0}\left|x-x_{0}\right|^{\sigma}
$$

for all $x \in \Omega$. If $1<p<n$, then there exists a positive constant $\lambda^{*}$ such that the problem (1.4) has a positive solution for any $\lambda \in\left(\lambda^{*}, \lambda_{1}\left(-\mathcal{L}_{p, a}\right)\right)$. 
Note that the point $x_{0}$ in the theorem above is a global minimum of $a(x)$. If further $x_{0}$ is unique, it suffices instead of assuming that $a(x) \geq a\left(x_{0}\right)+C_{0}\left|x-x_{0}\right|^{\sigma}$ around $x_{0}$.

We next present two main nonexistence results, the second one deals with lower order minimum points.

THEOREM 1.3. Assume that $\Omega$ is of $C^{1}$ class and star-shaped with respect to $x_{0} \in \Omega$, a is of $C^{1}$ class on $\bar{\Omega} \backslash\left\{x_{0}\right\}$ and $\nabla a(x) \cdot\left(x-x_{0}\right)$ extends continuously to $x_{0}$ and is nonnegative on $\Omega$. If $1<p<n$, then the problem (1.4) has no positive $C^{1}$ solution for any $\lambda \in(-\infty, 0]$.

It deserves mention that the problem (1.4) has no positive solution whenever $\lambda \geq \lambda_{1}\left(-\mathcal{L}_{p, a}\right)$. This assertion follows from a slight adaptation of the proof of Theorem 3.3 of Guedda and Veron [25].

The assumptions in the theorem above imply that $x_{0}$ is a global minimum point of $a(x)$, as can easily be checked.

THEOREM 1.4. Assume that $\Omega$ is of $C^{1}$ class and star-shaped with respect to $x_{0} \in \Omega$, a is of $C^{1}$ class on $\bar{\Omega} \backslash\left\{x_{0}\right\}$ and $\nabla a(x) \cdot\left(x-x_{0}\right)$ extends continuously to $x_{0}$. Assume further that there exist constants $0<\sigma \leq p$ and $C_{0}>0$ such that

$$
\nabla a(x) \cdot\left(x-x_{0}\right) \geq \sigma C_{0}\left|x-x_{0}\right|^{\sigma}
$$

for all $x \in \Omega$. If $1<p<n$, then there exists a positive constant $\lambda_{*}$ such that the problem (1.4) has no positive $C^{1}$ solution for any $\lambda \in\left(-\infty, \lambda_{*}\right]$.

A quite simple argument shows that the condition (1.8) implies (1.7). So, the conditions assumed in Theorems 1.2 and 1.4and standard regularity theorems guarantees the existence of constants $0<\lambda_{*} \leq \lambda^{*}$ such that a positive $C^{1}$ solution of (1.4) exists for any $\lambda \in\left(\lambda^{*}, \lambda_{1}\left(-\mathcal{L}_{p, a}\right)\right)$ and no existence occurs for any $\lambda \in\left(-\infty, \lambda_{*}\right]$. Regarding the case that $n \geq p^{2}$, $\Omega$ is star-shaped with respect to $x_{0}$ and $a(x)=1+\left|x-x_{0}\right|^{\sigma}$, we discover that the picture changes considerably according to the cases $\sigma>p$ to $\sigma \leq p$. Precisely, assuming $\Omega \in C^{1}$ and $\sigma>p$, Theorems 1.1 and 1.3 and the above-mentioned nonexistence result for $\lambda \geq \lambda_{1}\left(-\mathcal{L}_{p, a}\right)$ provide a positive $C^{1}$ solution for the problem (1.4) if, and only if, $\lambda \in\left(0, \lambda_{1}\left(-\mathcal{L}_{p, a}\right)\right)$. On the other hand, if $\sigma \leq p$, then a nonexistence range is given by $\left(-\infty, \lambda_{*}\right]$ for some positive number $\lambda_{*}$. Moreover, when $\sigma=p$ and $n=p^{2}$, we prove in Section 6 that $\lambda_{*}=\lambda^{*}$. In order to further exploit this example, we investigate what happens as $\sigma$ tends to zero. As we shall see later, $\lambda^{*}=0$ in the case that $\sigma=0$. So, in our approach, the classical BrezisNirenberg result for $p$-Laplace operators and $n \geq p^{2}$ is recovered, which can be framed in the cases $\sigma=0$ or $\sigma>p$. However, it is interesting to note that

$$
\liminf _{\sigma \rightarrow 0^{+}} \lambda^{*} \geq \lambda_{1}\left(-\Delta_{p}\right)>0 .
$$


In particular, $\lambda^{*}$ is not continuous at $\sigma=0$. All assertions concerning the diffusion term $a(x)=1+\left|x-x_{0}\right|^{\sigma}$ will be proved in Sections 5 and 6 .

In conclusion, if $\Omega$ is star-shaped with respect to any point (e.g. a ball), then the range of the values $\lambda$ for which the problem (1.4) has a positive solution relies heavily on the order of interior global minimum points of the diffusion $a(x)$.

An important advantage of Theorems 1.2 and 1.4 is that the constants $\lambda^{*}$ and $\lambda_{*}$ are constructed explicitly in terms of best constants associated to Euclidean Hardy-Sobolev type inequalities. In particular, we will be able to furnish upper and lower bounds for these constants, see Section 5 for more details.

In order to find solutions to problem (1.1) we can use either minimax methods involving the free energy functional or constrained minimization. We refer for instance to the Willem's book [38] for more details. Here we employ the second strategy. Precisely, consider the functional

$$
\Psi_{p, a}(u)=\int_{\Omega} a(x)|\nabla u|^{p} d x-\lambda \int_{\Omega}|u|^{p} d x
$$

constrained to the set $E_{p}:=\left\{u \in W_{0}^{1, p}(\Omega): \int_{\Omega}|u|^{p^{*}} d x=1\right\}$. Since $\Psi_{p, a}(u)=$ $\Psi_{p, a}(|u|)$ for all $u \in E_{p}$, the existence of a minimizer for $\Psi_{p, a \mid E_{p}}$ leads readily to a positive solution of (1.4).

As we shall see in Section 2, the condition (1.6) assumed in Theorem 1.1 combined with standard estimates on bubbles around $x_{0}$ yields

$$
\Psi_{p, a}\left(u_{0}\right)<m_{a} K(n, p)^{-p}
$$

on some function $u_{0} \in E_{p}$, where $m_{a}$ denotes the minimum value of $a(x)$ on $\bar{\Omega}$ and $K(n, p)$ is the best constant to the embedding of $W_{0}^{1, p}(\Omega)$ into $L^{p^{*}}(\Omega)$. Once this is done, the existence of a positive solution is classically deduced from the preceding estimate. We present a complete proof for convenience of the reader. A different proof can also be deduced by using the Ekeland variational principle and a compactness result recently obtained by Mercuri and Willem in [30].

The proof of Theorem 1.2 involves the validity on $C_{0}^{\infty}(\Omega)$ of the sharp Sobolev inequality

$$
\left(\int_{\Omega}|u|^{p^{*}} d x\right)^{p / p^{*}} \leq K(n, p)^{p} \int_{\Omega}|\nabla u|^{p} d x
$$

and of the sharp Hardy-Sobolev inequality

$$
\int_{\Omega}|u|^{p} d x \leq K_{0}\left(n, p, \sigma, \Omega, x_{0}\right)^{p} \int_{\Omega}\left|x-x_{0}\right|^{\sigma}|\nabla u|^{p} d x
$$

provided that $0<\sigma \leq p$. Inequality (1.10) is a special case of the famous Caffarelli-Konh-Nirenberg inequality presented in [10]. When $\sigma=p$, the value of $K_{0}\left(n, p, \sigma, \Omega, x_{0}\right)$ does not depend on $\Omega$ and $x_{0}$, namely $K_{0}\left(n, p, \sigma, \Omega, x_{0}\right)=p / n$, as can be seen in [27] for $p=2$ and in [1] for $p \neq 2$. Thanks to (1.9) and (1.10) 
and to the condition (1.7), the positive constant $\lambda^{*}$ will be constructed as the supremum of values $\lambda>0$ for which the inequality

$$
\left(\int_{\Omega}|u|^{p^{*}} d x\right)^{p / p^{*}}+\lambda m_{a}^{-1} K(n, p)^{p} \int_{\Omega}|u|^{p} d x \leq m_{a}^{-1} K(n, p)^{p} \int_{\Omega} a(x)|\nabla u|^{p} d x
$$

holds for all $u \in C_{0}^{\infty}(\Omega)$. Section 3 will be devoted to the detailed proof of Theorems 1.1 and 1.2.

Pohožaev type integral identities are powerful tools in proving nonexistence of solutions for quasilinear elliptic boundary problems. Related to Theorem 1.3, we use one of them, namely a version of the Pucci-Serrin identity [34] for $C^{2}$ solutions refined by Degiovanni, Musesti and Squassina in [15] for $C^{1}$ solutions. The same identity is useful in the proof of Theorem 1.4 together with the sharp Hardy-Sobolev inequality (1.10) which plays a key role. The proof of Theorems 1.3 and 1.4 will be presented in Section 4. In Section 5, upper and lower bounds will be obtained for $\lambda^{*}$ and $\lambda_{*}$ considering some particularly interesting examples of diffusion coefficients $a(x)$. In Section 6 , it will be outlined some open problems which are naturally motivated by our results. Part of the tools required in proofs will be provided in Section 2 .

\section{Preliminary tools}

Our aim in this section is to provide two suitable lemmas for the proof of the main theorems.

As mentioned in the introduction, nontrivial solutions of (1.4) can be found as critical points of the functional

$$
\Psi_{p, a}(u)=\int_{\Omega} a(x)|\nabla u|^{p} d x-\lambda \int_{\Omega}|u|^{p} d x
$$

constrained to the set $E_{p}:=\left\{u \in W_{0}^{1, p}(\Omega): \int_{\Omega}|u|^{p^{*}} d x=1\right\}$. Such a critical point is called a least energy solution of (1.4) and

$$
c_{p, a}:=\inf _{u \in E_{p}} \Psi_{p, a}(u)
$$

is called the least energy level of $\Psi_{p, a}$ on $E_{p}$.

The conclusion of Theorems 1.1 and 1.2 relies on a rather standard lemma whose proof will be outlined for reader's convenience. The proof given below follows the ideas of an elegant and simple proof of minimizers existence presented by Barstch and Willem in [4] and communicated to us by M. Willem.

Given a positive continuous function $a(x)$ on $\bar{\Omega}$, recall that $m_{a}$ denotes its minimum value on $\bar{\Omega}$ and $K(n, p)$ denotes the best constant to the embedding of $W_{0}^{1, p}(\Omega)$ into $L^{p^{*}}(\Omega)$. 
LEMMA 2.1. If $0<c_{p, a}<m_{a} K(n, p)^{-p}$, then the constrained functional $\left.\Psi_{p, a}\right|_{E_{p}}$ attains its infimum.

Proof. Let $\left(u_{j}\right) \subset E_{p}$ be such that

$$
\Psi_{p, a}\left(u_{j}\right)=\int_{\Omega} a(x)\left|\nabla u_{j}\right|^{p} d x-\lambda \int_{\Omega}\left|u_{j}\right|^{p} d x \rightarrow c_{p, a}
$$

as $j \rightarrow+\infty$. We can assume that $u_{j} \rightarrow u$ in $W_{0}^{1, p}(\Omega), u_{j} \rightarrow u$ in $L^{p}(\Omega)$ and $u_{j} \rightarrow u$ almost everywhere. on $\Omega$. By the sharp Sobolev inequality,

$$
c_{p, a}=\lim _{j \rightarrow+\infty}\left(\int_{\Omega} a(x)\left|\nabla u_{j}\right|^{p} d x-\lambda \int_{\Omega}\left|u_{j}\right|^{p} d x\right) \geq m_{a} K(n, p)^{-p}-\lambda \int_{\Omega}|u|^{p} d x .
$$

Thus, since $c_{p, a}<m_{a} K(n, p)^{-p}$, it follows that $u \neq 0$. We now show that $\|u\|_{L^{p^{*}}(\Omega)}=1$. As in [4], consider, for $h>0$, the auxiliary functions

$$
T_{h}(s)=\min (\max (s,-h), h) \quad \text { and } \quad R_{h}(s)=s-T_{h}(s) .
$$

As can be easily checked, $T_{h}(u), R_{h}(u) \in W_{0}^{1, p}(\Omega)$ and

$$
\int_{\Omega} a(x)|\nabla u|^{p} d x=\int_{\Omega} a(x)\left|\nabla T_{h}(u)\right|^{p} d x+\int_{\Omega} a(x)\left|\nabla R_{h}(u)\right|^{p} d x
$$

for any $u \in W_{0}^{1, p}(\Omega)$. Consequently,

$$
\begin{aligned}
c_{p, a}= & \lim _{j \rightarrow+\infty}\left(\int_{\Omega} a(x)\left|\nabla u_{j}\right|^{p} d x-\lambda \int_{\Omega}\left|u_{j}\right|^{p} d x\right) \\
= & \lim _{j \rightarrow+\infty}\left(\int_{\Omega} a(x)\left|\nabla T_{h}\left(u_{j}\right)\right|^{p} d x+\int_{\Omega} a(x)\left|\nabla R_{h}\left(u_{j}\right)\right|^{p} d x\right)-\lambda \int_{\Omega}|u|^{p} d x \\
= & \lim _{j \rightarrow+\infty}\left(\int_{\Omega} a(x)\left|\nabla T_{h}\left(u_{j}\right)\right|^{p} d x\right. \\
& \left.-\lambda \int_{\Omega}\left|T_{h}\left(u_{j}\right)\right|^{p} d x+\int_{\Omega} a(x)\left|\nabla T_{h}\left(u_{j}\right)\right|^{p} d x-\lambda \int_{\Omega}\left|R_{h}\left(u_{j}\right)\right|^{p} d x\right) \\
& +\lambda \int_{\Omega}\left|T_{h}(u)\right|^{p} d x+\lambda \int_{\Omega}\left|R_{h}(u)\right|^{p} d x-\lambda \int_{\Omega}|u|^{p} d x \\
\geq & c_{p, a} \lim _{j \rightarrow+\infty}\left[\left(\int_{\Omega}\left|T_{h}\left(u_{j}\right)\right|^{p^{*}} d x\right)^{p / p^{*}}+\left(\int_{\Omega}\left|R_{h}\left(u_{j}\right)\right|^{p^{*}} d x\right)^{p / p^{*}}\right] \\
& +\lambda \int_{\Omega}\left|T_{h}(u)\right|^{p} d x+\lambda \int_{\Omega}\left|R_{h}(u)\right|^{p} d x-\lambda \int_{\Omega}|u|^{p} d x .
\end{aligned}
$$

Thanks to Lemma 3.1 of [4], one gets

$$
\begin{aligned}
c_{p, a} \geq & c_{p, a}\left[\left(\int_{\Omega}\left|T_{h}(u)\right|^{p^{*}} d x\right)^{p / p^{*}}+\left(1+\int_{\Omega}\left|R_{h}(u)\right|^{p^{*}} d x-\int_{\Omega}|u|^{p^{*}} d x\right)^{p / p^{*}}\right] \\
& +\lambda \int_{\Omega}\left|T_{h}(u)\right|^{p} d x+\lambda \int_{\Omega}\left|R_{h}(u)\right|^{p} d x-\lambda \int_{\Omega}|u|^{p} d x
\end{aligned}
$$


Using that $c_{p, a}>0$, when $h \rightarrow+\infty$, one obtains

$$
1 \geq\left(\int_{\Omega}|u|^{p^{*}} d x\right)^{p / p^{*}}+\left(1-\int_{\Omega}|u|^{p^{*}} d x\right)^{p / p^{*}}
$$

so that $\|u\|_{L^{*}(\Omega)}=1$. By lower semi-continuity, it then follows that $u$ is a minimizer for $c_{p, a}$.

As previously already quoted, nonexistence results for elliptic problems are often established through integral identities satisfied by any solution. Particularly, the proof of Theorems 1.3 and 1.4 relies on the following integral identity:

Lemma 2.2 ([15], [34]). Let $1<p<n$. Assume that $\Omega$ is of $C^{1}$ class, a is of $C^{1}$ class on $\bar{\Omega} \backslash\left\{x_{0}\right\}$ and $b(x)=\nabla a(x) \cdot\left(x-x_{0}\right)$ extends continuously to $x_{0}$. Let $u$ be a $C^{1}$ solution of (1.4). Then $u$ satisfies the identity

$$
\frac{1}{p} \int_{\partial \Omega} a(x)|\nabla u|^{p}\left(x-x_{0}\right) \cdot \nu d s=\lambda \int_{\Omega}|u|^{p} d x-\frac{1}{p} \int_{\Omega} b(x)|\nabla u|^{p} d x,
$$

where $\nu$ denotes the exterior normal field to $\partial \Omega$ and ds stands for the element of area corresponding to $\partial \Omega$.

\section{Proof of Theorems 1.1 and 1.2}

The proof of Theorems 1.1 and 1.2 involves Lemma 2.1, bubbles estimates around $x_{0}$ and the valid of the sharp Hardy-Sobolev inequality (1.10) on $C_{0}^{\infty}(\Omega)$.

Proof OF TheOrem 1.1. Although we could provide an alternative proof by using some ideas due to Egnell [18], [19], we choose considering another functional on $E$ defined in the introduction, namely $\Psi_{p, a}$, in a similar way to the one of Garcia Azorero and Peral Alonso [21]. As explained in detail in the introduction, the key point of this proof is Lemma 2.1. First, without loss of generality, assume that $x_{0}=0$. By the assumption (1.6), there exists $\delta>0$ such that $B_{\delta}(0) \subset \Omega$ and

$$
a(x) \leq a(0)+C_{0}|x|^{\sigma}
$$

for all $x \in B_{\delta}(0)$. Using this condition, we have

$$
\begin{aligned}
& c_{p, a} \leq \inf _{u \in W_{0}^{1, p}\left(B_{\delta}(0)\right) \backslash\{0\}} \frac{\int_{B_{\delta}(0)} a(x)|\nabla u|^{p} d x-\int_{B_{\delta}(0)} \lambda|u|^{p} d x}{\left(\int_{B_{\delta}(0)}|u|^{p^{*}} d x\right)^{p / p^{*}}} \\
& \leq \inf _{u \in W_{0}^{1, p}\left(B_{\delta}(0)\right) \backslash\{0\}} \frac{\int_{B_{\delta}(0)} a(0)|\nabla u|^{p} d x+C_{0} \int_{B_{\delta}(0)}|x|^{\sigma}|\nabla u|^{p} d x-\lambda \int_{B_{\delta}(0)}|u|^{p} d x}{\left(\int_{B_{\delta}(0)}|u|^{p^{*}} d x\right)^{p / p^{*}}} .
\end{aligned}
$$


In order to prove that $c_{p, a}<m_{a} K(n, p)^{-p}$, consider now a cutoff function $\eta \in$ $C_{0}^{\infty}\left(B_{\delta}(0)\right)$ with $\eta=1$ around 0 and extremal functions for $K(n, p)$ on $\mathcal{D}\left(\mathbb{R}^{n}\right)$ given to one-parameter $\varepsilon>0$ by

$$
v_{\varepsilon}(x)=c_{n, p} \varepsilon^{(n-p) / p(p-1)}\left(\varepsilon^{p /(p-1)}+|x|^{p /(p-1)}\right)^{(p-n) / p},
$$

where $c_{n, p}$ is normalized so that $\left\|v_{\varepsilon}\right\|_{p^{*}}=1$, see [3] or [37].

According to [21], $w_{\varepsilon}=\eta v_{\varepsilon} \in W_{0}^{1, p}\left(B_{\delta}(0)\right)$ and satisfies

$$
\begin{aligned}
\left\|\nabla w_{\varepsilon}\right\|_{p}^{p} & =K(n, 2)^{-p}+O\left(\varepsilon^{p+\left(n-p^{2}\right) /(p-1)}\right), \\
\left\|w_{\varepsilon}\right\|_{p^{*}}^{p} & =1+O\left(\varepsilon^{n}\right), \\
\left\|w_{\varepsilon}\right\|_{p}^{p} & = \begin{cases}a_{n, p} \varepsilon^{p}+O\left(\varepsilon^{p+\left(n-p^{2}\right) /(p-1)}\right) & \text { if } n>p^{2}, \\
a_{n, p} \varepsilon^{p}|\log \varepsilon|+O\left(\varepsilon^{p}\right) & \text { if } n=p^{2},\end{cases}
\end{aligned}
$$

where

$$
\begin{array}{ll}
a_{n, p}=c_{n, p}^{p} \omega_{n-1} \frac{(p-1) \Gamma\left(\frac{n-p^{2}}{p}\right) \Gamma\left(\frac{n p-n}{p}\right)}{p \Gamma(n-p)} & \text { for } n>p^{2}, \\
a_{n, p}=c_{n, p}^{p} \omega_{n-1} & \text { for } n=p^{2} .
\end{array}
$$

Here $\omega_{n-1}$ stands for the area of the round unit sphere $S^{n-1}$.

On the other hand, when $\sigma>p$, straightforward computations yield

$$
\int_{B_{\delta}(0)}|x|^{\sigma}\left|\nabla w_{\varepsilon}\right|^{p} d x= \begin{cases}O\left(\varepsilon^{\sigma}\right) & \text { if } \sigma<\frac{n-p}{p-1} \\ O\left(\varepsilon^{\sigma}|\log \varepsilon|\right) & \text { if } \sigma=\frac{n-p}{p-1} \\ O\left(\varepsilon^{(n-p) /(p-1)}\right) & \text { if } \sigma>\frac{n-p}{p-1} .\end{cases}
$$

In particular,

$$
\int_{B_{\delta}(0)}|x|^{\sigma}\left|\nabla w_{\varepsilon}\right|^{p} d x= \begin{cases}o\left(\varepsilon^{p}\right) & \text { if } n>p^{2} \\ O\left(\varepsilon^{p}\right) & \text { if } n=p^{2}\end{cases}
$$

In conclusion, a combination between (3.1) and (3.2) produces, for $n \geq p^{2}$ and $\lambda>0$,

$$
c_{p, a} \leq \frac{K(n, p)^{-p}-a_{n, p} \lambda \varepsilon^{p}+o\left(\varepsilon^{p}\right)}{m_{a}^{-1}\left(1+O\left(\varepsilon^{n}\right)\right)}<m_{a} K(n, p)^{-p}
$$

provided $\varepsilon>0$ is small enough.

For $n=p^{2}$ and $\lambda>0$, again we have

$$
c_{p, a} \leq \frac{K(n, p)^{-p}-a_{n, p} \lambda \varepsilon^{p}|\log \varepsilon|+O\left(\varepsilon^{p}\right)}{m_{a}^{-1}\left(1+O\left(\varepsilon^{n}\right)\right)}<m_{a} K(n, p)^{-p}
$$

for $\varepsilon>0$ small too. 
By Lemma 2.1, the constrained functional $\left.\Psi_{p, a}\right|_{E_{p}}$ admits a minimizer $u$ which can be taken nonnegative. In particular, $u$ satisfies for some Lagrange multiplier $\mu \in \mathbb{R}$,

$$
\begin{cases}-\mathcal{L}_{p, a} u=\mu u^{p^{*}-1}+\lambda u^{p-1} & \text { in } \Omega \\ u=0 & \text { on } \partial \Omega .\end{cases}
$$

Since $u \in E_{p}$, we have $\mu=\Psi_{p, a}(u)$. Using now that $\lambda<\lambda_{1}\left(-\mathcal{L}_{p, a}\right)$, one obtains $\mu>0$. Finally, one easily checks that $\mu^{1 /\left(p^{*}-p\right)} u$ is a positive solution of (1.4).

If the condition $a(x)=a\left(x_{0}\right)+o\left(\left|x-x_{0}\right|^{p}\right)$ is assumed around $x_{0}$, then (3.2) is replaced by

$$
\int_{\Omega}\left|x-x_{0}\right|^{p}\left|\nabla w_{\varepsilon}\right|^{p} d x= \begin{cases}b \varepsilon^{p}+o\left(\varepsilon^{p}\right) & \text { if } n>p^{2}, \\ b \varepsilon^{p}|\log \varepsilon|+O\left(\varepsilon^{p}\right) & \text { if } n=p^{2},\end{cases}
$$

for constants $b>0$ small enough. The rest of the proof then follows.

Proof of Theorem 1.2. The building strategy for $\lambda^{*}$ changes when $0<$ $\sigma \leq p$ since estimates with bubbles has no effect. In this case, the validity of (1.10) is essential. Indeed, by (1.7) and (1.10) we can write

$$
\begin{aligned}
m_{a}^{-1} K(n, p)^{p} & \int_{\Omega} a(x)|\nabla u|^{p} d x \\
& \geq K(n, p)^{p} \int_{\Omega}|\nabla u|^{p} d x+C_{0} m_{a}^{-1} K(n, p)^{p} \int_{\Omega}\left|x-x_{0}\right|^{\sigma}|\nabla u|^{p} d x \\
& \geq\left(\int_{\Omega}|u|^{p^{*}} d x\right)^{p / p^{*}}+C_{0} K_{0}\left(n, p, \sigma, \Omega, x_{0}\right)^{-p} m_{a}^{-1} K(n, p)^{p} \int_{\Omega}|u|^{p} d x .
\end{aligned}
$$

Consider the set $\Theta$ of values $\lambda>0$ for which the inequality

$$
m_{a}^{-1} K(n, p)^{p} \int_{\Omega} a(x)|\nabla u|^{p} d x \geq\left(\int_{\Omega}|u|^{p^{*}} d x\right)^{p / p^{*}}+\lambda m_{a}^{-1} K(n, p)^{p} \int_{\Omega}|u|^{p} d x
$$

holds for all $u \in C_{0}^{\infty}(\Omega)$.

Remark that the set $\Theta$ is non-empty, since $C_{0} K_{0}\left(n, p, \sigma, \Omega, x_{0}\right)^{-p}$ belongs to $\Theta$. Let $\lambda^{*}:=\sup \Theta$. In particular, $\lambda^{*} \geq C_{0} K_{0}\left(n, p, \sigma, \Omega, x_{0}\right)^{-p}$. Note also that $\lambda^{*}$ is finite and $\Theta$ is a closed set, since the inequality

$$
\begin{aligned}
m_{a}^{-1} K(n, p)^{p} \int_{\Omega} a(x) \mid & \left.\nabla u\right|^{p} d x \\
& \geq\left(\int_{\Omega}|u|^{p^{*}} d x\right)^{p / p^{*}}+\lambda^{*} m_{a}^{-1} K(n, p)^{p} \int_{\Omega}|u|^{p} d x
\end{aligned}
$$

holds for all $u \in C_{0}^{\infty}(\Omega)$.

It now suffices to prove that $c_{p, a}<m_{a} K(n, p)^{-p}$ for any $\lambda \in\left(\lambda^{*},+\infty\right)$ and $\lambda^{*}<\lambda_{1}\left(-\mathcal{L}_{p, a}\right)$. The first assertion follows immediately from the definition 
of $\lambda^{*}$. In fact, since $\lambda \notin \Theta$ for any $\lambda>\lambda^{*}$, there exists $u_{0} \in C_{0}^{\infty}(\Omega)$ such that

$$
\begin{aligned}
m_{a}^{-1} K(n, p)^{p} \int_{\Omega} a(x) \mid & \left.\nabla u_{0}\right|^{p} d x \\
< & \left(\int_{\Omega}\left|u_{0}\right|^{p^{*}} d x\right)^{p / p^{*}}+\lambda m_{a}^{-1} K(n, p)^{p} \int_{\Omega}\left|u_{0}\right|^{p} d x
\end{aligned}
$$

or equivalently, $c_{p, a}<m_{a} K(n, p)^{-p}$. For the second one, we choose an eigenfunction $\varphi_{1}$ corresponding to the first eigenvalue $\lambda_{1}\left(-\mathcal{L}_{p, a}\right)$ of the operator $-\mathcal{L}_{p, a}$ as a test function in (3.3). So, we are led to

$$
\lambda_{1}\left(-\mathcal{L}_{p, a}\right) \int_{\Omega}\left|\varphi_{1}\right|^{p} d x=\int_{\Omega} a(x)\left|\nabla \varphi_{1}\right|^{p} d x>\lambda^{*} \int_{\Omega}\left|\varphi_{1}\right|^{p} d x,
$$

so that the assertion follows. Proceeding now exactly as in the previous proof, one derives a positive solution of (1.4) for any $\lambda \in\left(\lambda^{*}, \lambda_{1}\left(-\mathcal{L}_{p, a}\right)\right)$.

\section{Proof of Theorems 1.3 and 1.4}

The proof of Theorems 1.3 and 1.4 is based on the Pohožaev type integral identity (2.1) given in Lemma 2.2.

Proof of TheOrem 1.3. Assume, by contradiction, that (1.4) admits a positive $C^{1}$ solution $u$ and that, without loss of generality, $x_{0}=0$. By the Hopf lemma due to Pucci, Serrin and Zhou (Theorem 1' of [35]), the gradient of $u$ never vanishes on $\partial \Omega$. Since $\Omega$ is star-shaped with respect to the origin, $x \cdot \nu$ is nonnegative and non-identically zero on $\partial \Omega$. So, thanks to the positivity of the function $a$, the left-hand side of (2.1) is positive too. But, by assumption, we have $\nabla a(x) \cdot x \geq 0$ for all $x \in \Omega$ and, therefore, the equation (2.1) would be violated if $\lambda \leq 0$.

Proof of Theorem 1.4. Arguing as in the preceding proof and using the assumption $\nabla a(x) \cdot x \geq \sigma C_{0}|x|^{\sigma}$ for all $x \in \Omega$, the identity (2.1) yields

$$
\lambda \int_{\Omega}|u|^{p} d x>\frac{\sigma}{p} C_{0} \int_{\Omega}|x|^{\sigma}|\nabla u|^{p} d x .
$$

Using now that $0<\sigma \leq p$ and evoking the sharp Hardy-Sobolev inequality (1.10), one gets

$$
\lambda \int_{\Omega}|u|^{p} d x>\frac{\sigma}{p} C_{0} K_{0}(n, p, \sigma, \Omega, 0)^{-p} \int_{\Omega}|u|^{p} d x,
$$

so that $\lambda>\lambda_{*}=: \frac{\sigma}{p} C_{0} K_{0}(n, p, \sigma, \Omega, 0)^{-p}$. Consequently, for star-shaped domains about the origin, the problem (1.4) has no positive $C^{1}$ solution for any $\lambda \in$ $\left(-\infty, \lambda_{*}\right]$ 


\section{Bounds for $\lambda^{*}$ and $\lambda_{*}$}

This section deals mainly with upper and lower bounds for $\lambda^{*}$ and $\lambda_{*}$ for some interesting examples of non-uniformly elliptic operators $\mathcal{L}_{p, a}$ of the type $\operatorname{div}\left(a(x)|\nabla u|^{p-2} \nabla u\right)$.

We first focus on the constant $\lambda^{*}$ provided in Theorem 1.2. Let $x_{0} \in \Omega$ be a global minimum point of $a(x)$ and assume that the function $a(x)$ satisfies (1.7), namely there exist constants $0<\sigma \leq p$ and $C_{0}>0$ such that

$$
a(x) \geq a\left(x_{0}\right)+C_{0}\left|x-x_{0}\right|^{\sigma}
$$

for all $x \in \Omega$. The construction of $\lambda^{*}$ produces

$$
\lambda^{*} \geq C_{0} K_{0}\left(n, p, \sigma, \Omega, x_{0}\right)^{-p}
$$

We recall that when $\sigma=p$, the exact value of the best constant $K_{0}\left(n, p, \sigma, \Omega, x_{0}\right)$ associated to the Hardy-Sobolev inequality (1.10) is given by

$$
K_{0}\left(n, p, p, \Omega, x_{0}\right)=\frac{p}{n} .
$$

Unfortunately, the value of this optimal constant is not known for $0<\sigma<p$, although some rough bounds can easily be deduced. Since $\lambda^{*}<\lambda_{1}\left(-\mathcal{L}_{p, a}\right)$, upper bounds for $\lambda^{*}$ can be obtained explicitly in terms of $\Omega$. Nevertheless, when $\sigma=p$ and $a(x)$ also satisfies

$$
a(x) \leq a\left(x_{0}\right)+D_{0}\left|x-x_{0}\right|^{p}
$$

around $x_{0}$ for some constant $D_{0}>0$, then estimates on bubbles concentrated at $x_{0}$ readily lead to an upper bound for $\lambda^{*}$ depending only on the dimension $n$. In fact, for the bubbles $w_{\varepsilon}$ defined in the proof of Theorem 1.1, we have

$$
\int_{\Omega} a\left(x_{0}\right)\left|\nabla w_{\varepsilon}\right|^{p} d x=m_{a} K(n, p)^{-p}+O\left(\varepsilon^{p+\left(n-p^{2}\right) /(p-1)}\right)
$$

and

$$
\int_{\Omega}\left|x-x_{0}\right|^{p}\left|\nabla w_{\varepsilon}\right|^{p} d x= \begin{cases}b_{n, p} \varepsilon^{p}+o\left(\varepsilon^{p}\right) & \text { if } n>p^{2} \\ b_{n, p} \varepsilon^{p}|\log \varepsilon|+O\left(\varepsilon^{p}\right) & \text { if } n=p^{2}\end{cases}
$$

with

$$
\begin{array}{ll}
b_{n, p}=c_{n, p}^{p} \omega_{n-1}\left(\frac{n-p}{p-1}\right)^{p} \frac{(p-1) \Gamma\left(\frac{n-p^{2}}{p}\right) \Gamma\left(\frac{n p+p^{2}-n}{p}\right)}{p \Gamma(n)} & \text { for } n>p^{2}, \\
b_{n, p}=p^{p} c_{n, p}^{p} \omega_{n-1} & \text { for } n=p^{2} .
\end{array}
$$


Thus, choosing $u=w_{\varepsilon}$ in (3.3), using the inequality (5.3) and after the bubbles estimates (3.1), (5.4) and (5.5), we deduce that

$$
\lambda^{*} \leq D_{0} \frac{b_{n, p}}{a_{n, p}} .
$$

By contrast, note that the constant $\lambda_{*}$ provided in Theorem 1.4 appears in an explicit way. Precisely, assume that $\Omega$ is a star-shaped domain with respect to $x_{0} \in \Omega$ and the function $a(x)$ satisfies (1.8), precisely there exist constants $0<\sigma \leq p$ and $C_{0}>0$ such that

$$
\nabla a(x) \cdot\left(x-x_{0}\right) \geq \sigma C_{0}\left|x-x_{0}\right|^{\sigma}
$$

for all $x \in \Omega$. By definition,

$$
\lambda_{*}:=\frac{\sigma}{p} C_{0} K_{0}\left(n, p, \sigma, \Omega, x_{0}\right)^{-p} .
$$

On the other hand, the condition (1.8) implies (1.7), so that

$$
\lambda_{*}:=\frac{\sigma}{p} C_{0} K_{0}\left(n, p, \sigma, \Omega, x_{0}\right)^{-p} \leq \frac{\sigma}{p} \lambda^{*} .
$$

Before going further, it can easily be checked from Lemma 2.2 and (5.7) that $\lambda_{*}=0$ for $\sigma=0$ and

$$
\lim _{\sigma \rightarrow 0^{+}} \lambda_{*}=0
$$

for any star-shaped bounded $C^{1}$ domain $\Omega$.

We now derive the value of $\lambda^{*}$ for $\sigma=0$ and its asymptotic behavior for $\sigma$ near zero. Namely, $\lambda^{*}=0$ for $\sigma=0$ and, however,

$$
\liminf _{\sigma \rightarrow 0^{+}} \lambda^{*} \geq \lambda_{1}\left(-\Delta_{p}\right)>0
$$

When $\sigma=0$, the term of diffusion $a(x)=1+\left|x-x_{0}\right|^{\sigma}$ becomes the constant function $a(x)=2$, so that $m_{a}=2$. Thus, the Euclidean sharp Sobolev inequality (1.9) can be rewritten as

$$
\left(\int_{\Omega}|u|^{p^{*}} d x\right)^{p / p^{*}} \leq m_{a}^{-1} K(n, p)^{p} \int_{\Omega} a(x)|\nabla u|^{p} d x .
$$

In particular, we deduce that $\lambda^{*} \geq 0$. The reverse inequality follows from a direct combination between the estimates of bubbles (3.1) and the inequality (3.3). Finally, from (5.1), we have

$$
\liminf _{\sigma \rightarrow 0^{+}} \lambda^{*} \geq \liminf _{\sigma \rightarrow 0^{+}} K_{0}\left(n, p, \sigma, \Omega, x_{0}\right)^{-p} .
$$


It remains then to show that $\lim _{\sigma \rightarrow 0^{+}} K_{0}\left(n, p, \sigma, \Omega, x_{0}\right)^{-p}=\lambda_{1}\left(-\Delta_{p}\right)$. But the inequality

$$
\begin{aligned}
\int_{\Omega}|u|^{p} d x \leq K_{0}\left(n, p, \sigma, \Omega, x_{0}\right)^{p} & \int_{\Omega}\left|x-x_{0}\right|^{\sigma}|\nabla u|^{p} d x \\
& \leq K_{0}\left(n, p, \sigma, \Omega, x_{0}\right)^{p}(\operatorname{diam} \Omega)^{\sigma} \int_{\Omega}|\nabla u|^{p} d x
\end{aligned}
$$

implies $\limsup _{\sigma \rightarrow 0^{+}} K_{0}\left(n, p, \sigma, \Omega, x_{0}\right)^{-p} \leq \lambda_{1}\left(-\Delta_{p}\right)$.

In order to achieve the desired limit, we evoke a result of [39] which asserts the existence, for each $0<\sigma<p$, of a weak solution $\varphi_{\sigma} \in W_{0, \sigma}^{1, p}(\Omega)$ of the problem

$$
\begin{cases}-\operatorname{div}\left(\left|x-x_{0}\right|^{\sigma}|\nabla u|^{p-2} \nabla u\right)=K_{0}\left(n, p, \sigma, \Omega, x_{0}\right)^{-p}|u|^{p-2} u & \text { in } \Omega \\ u=0 & \text { on } \partial \Omega\end{cases}
$$

with $\left\|\varphi_{\sigma}\right\|_{L^{p}(\Omega)}=1$, where $W_{0, \sigma}^{1, p}(\Omega)$ denotes the completion of $C_{0}^{1}(\Omega)$ under the norm

$$
\|\left. u\right|_{W_{0, \sigma}^{1, p}}:=\left(\int_{\Omega}\left|x-x_{0}\right|^{\sigma}|\nabla u|^{p} d x\right)^{1 / p}
$$

According to the proof of Theorem 1.4 of [12], namely pages 673 and 674 , up to a subsequence, $\nabla \varphi_{\sigma}$ converges to $\nabla \varphi$ almost everywhere, where $\varphi \in W_{\mathrm{loc}}^{1, p}(\Omega \backslash$ $\left.\left\{x_{0}\right\}\right)$. Probably, an alternative proof of this convergence can be given by using the ideas of [14]. For a fixed number $0<\gamma<p$, there exist constants $c_{0}, c_{1}>0$, independent of $\sigma$, such that

$$
\begin{aligned}
& \int_{\Omega}\left|x-x_{0}\right|^{\gamma}\left|\nabla \varphi_{\sigma}\right|^{p} d x \leq c_{0} \int_{\Omega}\left|x-x_{0}\right|^{\sigma}\left|\nabla \varphi_{\sigma}\right|^{p} d x \\
& \quad=c_{0} K_{0}\left(n, p, \sigma, \Omega, x_{0}\right)^{-p} \int_{\Omega} \varphi_{\sigma}^{p} d x=K_{0}\left(n, p, \sigma, \Omega, x_{0}\right)^{-p} \leq c_{1}
\end{aligned}
$$

for all $0<\sigma<\gamma$. In particular, the sequence $\left(\varphi_{\sigma}\right)$ is bounded in $W_{0, \gamma}^{1, p}(\Omega)$ and then, by a natural extension of Lemma 2.1 of [13] to $p \neq 2$, namely Theorem 2.1 of [39], $\varphi_{\sigma}$ converges, modulo a subsequence, to $\varphi$ in $L^{p}(\Omega)$, so that $\|\varphi\|_{L^{p}(\Omega)}=1$. Finally, Fatou's lemma yields

$$
\begin{aligned}
\int_{\Omega}|\nabla \varphi|^{p} d x \leq \liminf _{\sigma \rightarrow 0^{+}} \int_{\Omega}\left|x-x_{0}\right|^{\sigma}\left|\nabla \varphi_{\sigma}\right|^{p} d x \\
\quad=\liminf _{\sigma \rightarrow 0^{+}} K_{0}\left(n, p, \sigma, \Omega, x_{0}\right)^{-p} \int_{\Omega}\left|\varphi_{\sigma}\right|^{p} d x=\liminf _{\sigma \rightarrow 0^{+}} K_{0}\left(n, p, \sigma, \Omega, x_{0}\right)^{-p},
\end{aligned}
$$

so that

$$
\liminf _{\sigma \rightarrow 0^{+}} K_{0}\left(n, p, \sigma, \Omega, x_{0}\right)^{-p} \geq \lambda_{1}\left(-\Delta_{p}\right) .
$$




\section{Open related problems}

Motivated by the discussion done in the previous section on bounds of $\lambda^{*}$ and $\lambda_{*}$, it naturally arises some interesting issues. First of all, under the condition (1.8), we saw that

$$
\lambda_{*}:=\frac{\sigma}{p} C_{0} K_{0}\left(n, p, \sigma, \Omega, x_{0}\right)^{-p} \leq \frac{\sigma}{p} \lambda^{*} .
$$

In particular, this inequality produces a gap whenever $0<\sigma<p$. Besides, when $\sigma=p$, one has $\lambda_{*}=:\left(n^{p} / p^{p}\right) C_{0} \leq \lambda^{*}$. So, two interesting questions arises under the condition (1.8):

(a) what happens when $\lambda \in\left(\lambda_{*}, \lambda^{*}\right]$ in the case $0<\sigma<p$;

(b) whether or not $\lambda_{*}$ is equal to $\lambda^{*}$ in the case $\sigma=p$.

The question (b) is affirmative for $n=p^{2}$ under the condition (1.8) with equality or, equivalently,

$$
a(x)=a\left(x_{0}\right)+C_{0}\left|x-x_{0}\right|^{p} \quad \text { in } \Omega .
$$

Precisely, when $n=p^{2}$, one has $b_{n, p} / a_{n, p}=p^{p}$ and, thanks to (5.6), the equality $\lambda_{*}=\lambda^{*}$ follows. This fact was established by Egnell [18] in the case $p=2$. On the other hand, when $n>p^{2}$, one easily checks that

$$
\lambda_{*}:=C_{0} \frac{n^{p}}{p^{p}}<C_{0} \frac{b_{n, p}}{a_{n, p}} .
$$

Surely it would be interesting to understand (a) and (b) in the apparently simpler situation $a(x)=1+\left|x-x_{0}\right|^{\sigma}$ with $x_{0} \in \Omega$. In this example, the number $\lambda^{*}$ corresponds to the optimal constant $\lambda$ associated to the weighted Sobolev inequality

$$
\left(\int_{\Omega}|u|^{p^{*}} d x\right)^{p / p^{*}}+\lambda K(n, p)^{p} \int_{\Omega}|u|^{p} d x \leq K(n, p)^{p} \int_{\Omega}\left(1+\left|x-x_{0}\right|^{\sigma}\right)|\nabla u|^{p} d x .
$$

Summarizing, we have $\lambda_{*}<\lambda^{*}$ whenever $0<\sigma<p, \lambda_{*}=(n / p)^{p} \leq \lambda^{*}$ when $\sigma=p$ and, by last, $\lambda_{*}=\lambda^{*}$ when $\sigma=p$ and $n=p^{2}$.

Perhaps the question (b) can be answered from a view point of optimal constants which consists in knowing the dependence of $\lambda^{*}$ on $\Omega$ and $x_{0}$ and, better yet, in determining its exact value. Still on the example $a(x)=1+\left|x-x_{0}\right|^{\sigma}$, a related question is to understand what occurs if $x_{0}$ does not belong to $\Omega$. This is the case when its minimum value is achieved on the boundary of $\Omega$.

Another particularly interesting example corresponding to $\sigma=p$ is

$$
a(x)=a\left(x_{0}\right)+\left(\left(x-x_{0}\right)^{t} B\left(x-x_{0}\right)\right)^{p / 2} \text { on } \Omega,
$$

where $B$ is a positive definite symmetric matrix. In this case,

$$
\lambda_{*}=K_{0}\left(n, p, B, \Omega, x_{0}\right)^{-p} \leq \lambda^{*},
$$


where $K_{0}\left(n, p, B, \Omega, x_{0}\right)^{p}$ is the best constant on $C_{0}^{\infty}(\Omega)$ to

$$
\int_{\Omega}|u|^{p} d x \leq K_{0}\left(n, p, B, \Omega, x_{0}\right)^{p} \int_{\Omega}\left(\left(x-x_{0}\right)^{t} B\left(x-x_{0}\right)\right)^{p / 2}|\nabla u|^{p} d x,
$$

and no answer to the question (b) is known even in dimension $n=p^{2}$.

Finally, concerning with operators $\mathcal{L}_{p, a}$, it would be important to know what happens if $a(x)$ has a behavior around $x_{0}$ distinct of those described in (1.6), (1.7) and (1.8). In particular, it may occur that the function $a(x)$ behaves differently around $x_{0}$ on each one direction of $\mathbb{R}^{n}$.

Acknowledgments. The second author was partially supported by CNPq and Fapemig. The authors are indebted to the referee for his valuable comments concerning this work.

\section{REFERENCES}

[1] B. Abdellaoui, E. Colorado and I. Peral, Some improved Caffarelli-Kohn-Nirenberg inequalities, Calc. Var. Partial Differential Equations 23 (2005), 327-345.

[2] G. Arioli and F. Gazzola, Some results on p-Laplace equations with a critical growth term, Differential Integral Equations 11 (1998), 311-326.

[3] T. Aubin, Problèmes isopérimétriques et espaces de Sobolev, J. Differential Geom. 11 (1976), 573-598.

[4] T. Bartsch and M. Willem, Some critical minimization problems for functions of bounded variations, J. Funct. Anal. 259 (2010), 3025-3035.

[5] V. Benci And G. Cerami, Existence of positive solutions of the equation $-\Delta u+a(x) u=$ $u^{(N+2) /(N-2)} \in \mathbb{R}^{N}$, J. Funct. Anal. 88 (1990), 90-117.

[6] I. Birindelli and F. Demengel, Existence of solutions for semilinear equations involving the p-Laplacian: the non coercive case, Calc. Var. Partial Differential Equations 20 (2004), 343-366.

[7] H. Brezis, Some variational problems with lack of compactness, Nonlinear Functional Analysis and its Applications (F. Browder, ed.), Berkeley, 1983, Proc. Symp. Pure Math., vol. 45, Amer. Math. Soc., 1986, pp. 165-201.

[8] H. Brezis And E. Lieb, Sobolev inequalities with remainder terms, J. Funct. Anal. 62 (1985), 73-86.

[9] H. Brezis And L. Nirenberg, Positive solutions of nonlinear elliptic equations involving critical Sobolev exponents, Comm. Pure Appl. Math. 36 (1983), 437-477.

[10] L. Caffarelli, R. Kohn and L. Nirenberg, First order interpolation inequalities with weights, Compositio Mathematica 53 (1984), 259-275.

[11] A. Capozzi, D. Fortunato and G. Palmieri, An existence result for nonlinear elliptic problems involving critical Sobolev exponent, Ann. Inst. H. Poincaré Anal. Non Linéaire 2 (1985), 463-470..

[12] J. CecCon And M. Montenegro, Compactness results for divergence type nonlinear elliptic equations, Ann. Mat. Pura Appl. 188 (2009), 653-677.

[13] K.S. Chou And C.W. Chu, On the best constant for a weighted Sobolev-Hardy inequality, J. London Math. Soc. 2 (1993), 137-151.

[14] S. De Valeriola and M. Willem, On some quasilinear elliptic problems, Adv. Nonlinear Stud. 9 (2009), 825-836. 
[15] M. Degiovanni And S. Lancelotti, Linking solutions for p-Laplace equations with nonlinearity at critical growth, J. Funct. Anal. 256 (2009), 3643-3659.

[16] M. Degiovanni, A. Musesti And M. Squassina, On the regularity of solutions in the Pucci-Serrin identity, Calculus of Variations and PDEs 18 (2003), 317-334.

[17] P. Drábek And S. Pohozaev, Positive solutions for the p-Laplacian: application of the fibering method, Proc. Roy. Soc. Edinburgh Sect. A 127 (1997), 703-726.

[18] H. Egnell, Semilinear elliptic equations involving critical Sobolev exponents, Arch. Rational Mech. Anal. 104 (1988), 27-56.

[19] Existence and nonexistence results for $m$-Laplace equations involving critical Sobolev exponents, Arch. Rational Mech. Anal. 104 (1988), 57-77.

[20] J.F. Escobar, Positive solutions for some semilinear elliptic equations with critical Sobolev exponents, Comm. Pure Appl. Math. 40 (1987), 623-657.

[21] J. Garcia Azorero And I. Peral Alonso, Existence and nonuniqueness for the p-Laplacian: nonlinear eigenvalues, Comm. Partial Differential Equations 12 (1987), 1389-1430.

[22] F. Gazzola AND B. RuF, Lower-order perturbations of critical growth nonlinearities in semilinear elliptic equations, Adv. Differential Equations 2 (1997), 555-572.

[23] Y. Ge, M. Musso And A. Pistoia, Sign changing tower of bubbles for an elliptic problem at the critical exponent in pierced non-symmetric domains, Comm. Partial Differential Equations 35 (2010), 1419-1457.

[24] M. Grossi, Radial solutions for the Brezis-Nirenberg problem involving large nonlinearities, J. Funct. Anal. 254 (2008), 2995-3036.

[25] M. Guedda And L. VÉRon, Quasilinear elliptic equations involving critical Sobolev exponents, Nonlinear Anal. 13 (1989), 879-902.

[26] R. Hadiji And H. Yazidi, Problem with critical Sobolev exponent and with weight, Chin. Ann. Math. Ser. B 28 (2007), 327-352.

[27] G.H. Hardy, J.E. Littlewood and G. Pólya, Inequalities, Cambridge University Press (1934).

[28] I. IL'yasov, On positive solutions of indefinite elliptic equations, C.R. Acad. Sci. Paris 332 (2001), 1-6.

[29] P.L. Lions, The concentration-compactness principle in the calculus of variations. The limit case. Part I, Rev. Mat. Iberoamericano 1.1 (1985), 145-201.

[30] C. Mercuri And M. Willem, A global compactness result for the p-Laplacian involving critical nonlinearities, Discrete Contin. Dynam. Syst. A 28 (2010), 469-493.

[31] M. Montenegro And R.J. Moura, On the influence of second order uniformly elliptic operators in nonlinear problems, Math. Nachr. (to appear).

[32] T. Ouyang, Positive solutions of semilinear elliptic equation $\Delta u+h u^{(n+2) /(n-2)}=0$, J. Differential Equations 123 (1995), 230-259.

[33] D. Passaseo, Some sufficient conditions for the existence of positive solutions to the equation $-\Delta u+a(x) u=u^{2^{*}-1}$ in bounded domains, Ann. Inst. H. Poincaré Anal. Non Linéaire 13 (1996), 185-227.

[34] P. PuCCi And J. Serrin, A general variational identity, Indiana Univ. J. 35 (1986), 681-703.

[35] P. PuCCi, J. Serrin And H. Zou, A strong maximum principle and a compact support principle for singular elliptic inequalities, J. Math. Pures Appl. 78 (1999), 769-789.

[36] M. Struwe, Variational Methods and Applications to Nonlinear Partial Differential Equations and Hamiltonian Systems, Springer-Verlag, Berlin, 1990.

[37] G. Talenti, Best constant in Sobolev inequality, Ann. Mat. Pura Appl. (4) 110 (1976), 353-372. 
[38] M. Willem, Minimax Theorems, Progress in Nonlinear Differential Equations and their Applications, Birhäuser, 1996.

[39] B. XUAN, The solvability of quasilinear Brezis-Nirenberg-type problems with singular weights, Nonlinear Anal. 62 (2005), 703-725.

[40] A priori estimate for a family of semi-linear elliptic equations with critical nonlinearity, J. Differential Equations 247 (2009), 1334-1353.

Renato José de Moura

Departamento de Matemática

Universidade Federal de São Carlos

13565-905, São Carlos, SP, BRAZIL

E-mail address: moura@dm.ufscar.br

Marcos Montenegro

Departamento de Matemática

Universidade Federal de Minas Gerais

Caixa Postal 702

30123-970, Belo Horizonte, MG, BRAZIL

E-mail address: montene@mat.ufmg.br 